\title{
The Iron Project and TIPTOPbase: Atomic Data and Opacities for Astrophysics
}

\author{
Sultana N. Nahar (The Iron Project Team) \\ Department of Astronomy, The Ohio State University, Columbus, $\mathrm{OH}$ \\ 43210, USA
}

\begin{abstract}
The Iron Project (IP) is an extension of the Opacity Project, an international collaboration of atomic physicists and astrophysicists. The aim of the IP is the development and application of new methods in theoretical atomic physics to the calculation of large-scale atomic data of high accuracy for radiative and collisional processes for astrophysically important elements.
\end{abstract}

The calculations are carried out using the close coupling $\mathrm{R}$-matrix method. The atomic processes of interest are: electron impact excitation, photoionization, bound-bound transitions, and electron-ion recombination. The primary emphasis of the IP (Hummer et al. 1993) is on calculating the excitation collision strengths for the iron peak elements, in particular $\mathrm{Fe}$ in all ionization stages. Relativistic effects are included through the Breit-Pauli R-matrix (BPRM) method for most of the radiative data. Current IP series publication list in Astronomy and Astrophysics totals to about 50 papers. The IP website is maintained at: www.usm.uni-muenchen.de/people/ip/iron-project.html

Results from the IP team are being archived in an interactive electronic database TIPTOPbase installed at two dedicated sites: NASA GSFC/HEARC (Goddard Space Flight Center/High Energy Astrophysics Research Center), and at the Center de Donnees Astronomiques de Strasbourg (CDS). The current database TOPbase (Cunto et al. 1993) accessible at NASA (http://heasarc.gsfc. nasa.gov) and at CDS (http://vizier.u-strasbg.fr/OP.html) contains data from the OP (1995, 1996): (i) 52,886 identified energy levels, (ii) 1,607,933 oscillator strengths, (iii) $\sim 100,000$ photoionization cross sections for ground \& excited states, and (v) monochromatic opacities (at CDS only). It also provides effective quantum numbers, radiative lifetimes, \& atomic constants and facilities for data manipulation, graphics capability, search engines. TIPTOPbase consitutes a major upgrade of TOPbase.

TIPTOPbase will contain (i) electron impact excitation cross sections and rate coefficients for all iron ions Fe I - XXVI, Ni III from the IP, (ii) A-values for transitions in the target ion, (iii) all radiative data from $\mathrm{TOPbase}$, (iv) new radiative data for all ionization stages of: $\mathrm{P}, \mathrm{Cl}, \mathrm{K}, \mathrm{Ni}$ II (not originally computed under the OP), (v) updated sets of radiative data, especially for Fe ions, from repeated CC calculations, (vi) "tail" photoionization cross sections at high energies that include the inner shell ionization, (vii) photoionization cross sections for fine structure levels including relativistic effects for highly charged ions, (viii) unified $(e+$ ion) recombination rate coefficients including radiative plus dielectronic recombination (level-specific \& total), (ix) fine structure f-values in- 
cluding relativistic effects for Xray/ UV/ O/ IR transitions, (x) additional data for $f$-values including inner-shell excitations in iron ions Fe VIII - XIII ("PLUS" data) calculated with SUPERSTRUCTURE, (xi) radiative accelerations.

In addition to atomic data, TIPTOPbase will enable on-line computation of 'customized' stellar opacities for the Rosseland mean and radiative forces for user-specified mixture of elements (Seaton 1997) using the monochromatic opacities $\kappa_{\nu}$ obtained under the OP assuming LTE for 17 elements: $\mathrm{H}, \mathrm{He}, \mathrm{C}$, $\mathrm{N}, \mathrm{O}, \mathrm{Ne}, \mathrm{Na}, \mathrm{Mg}, \mathrm{Al}, \mathrm{Si}, \mathrm{S}, \mathrm{Ar}, \mathrm{Ca}, \mathrm{Cr}, \mathrm{Mn}, \mathrm{Fe}$ and $\mathrm{Ni}$ (Seaton et al 1994). Tables of $\kappa_{\nu}$ have been archived as function of $\log (u=h \nu / k T)$ at a mesh of $\left(\mathrm{T}, \mathrm{N}_{e}\right)$, where $\mathrm{T}$ is the temperature and $\mathrm{N}_{e}$ is the electron density.

The Rosseland mean opacities $\kappa_{R}$ defined by,

$$
\frac{1}{\kappa_{R}}=\frac{\int_{0}^{\infty} \frac{1}{\kappa_{\nu}} g(u) d u}{\int_{0}^{\infty} g(u) d u}
$$

where $\mathrm{u}=\mathrm{h} \nu / \mathrm{kT}$ and $\mathrm{g}(\mathrm{u})$ is the Planck weighting function (corrected for stimulated emission), $g(u)=15 /\left(4 \pi^{4}\right) u^{4} e^{-u}\left(1-e^{-u}\right)^{-2}$, are obtained for chemical compositions with different mass fractions for $\mathrm{H}(\mathrm{X}), \mathrm{He}(\mathrm{Y})$ and metals $(\mathrm{Z})$, such that $\mathrm{X}+\mathrm{Y}+\mathrm{Z}=1$. Rosseland means $\kappa_{R}(T, \rho)$, where $\rho$ is the mass density $(\mathrm{g} / \mathrm{cc}$ ) have been tabulated with standard solar and non-solar abundances. Also tabulated are partial derivatives of $\kappa_{R}(T, \rho),\left[\partial \log \left(\kappa_{R}\right) / \partial \log (T)\right]_{\rho}$, $\left[\partial \log \left(\kappa_{R}\right) / \partial \log (\rho)\right]_{T}$, required for stellar structure and pulsation studies.

TIPTOPbase will also include radiative accelaration, $\mathrm{g}_{\text {rad }}(\mathrm{k})$, recently used to estimte the diffusion of iron-group elements (Seaton 1999). Radiative diffusion can lead to large changes in abundances of individual elements in a star as gravitational forces are counteracted by radiative levitation. Diffusion calculations require $\mathrm{g}_{\text {rad }}(\mathrm{k})$ at all depth points in a star, as a function of abundance of element k (Seaton 1997),

$$
g_{\text {rad }}(k)=(1 / c) \int \sigma_{\nu}(k) F_{\nu} d \nu / M(k),
$$

where $\mathrm{M}(\mathrm{k})$ is the mass of atom $\mathrm{k}$ and the flux $\mathrm{F}=\int F_{\nu} d \nu$ is related to the Rosseland mean $\kappa_{R}$ as $F_{\nu}=\left(\kappa_{R} / \kappa_{\nu}\right)(h / k T) g(u)$. Interpolation codes will be provided in TIPTOPbase to obtain radiative accelerations for user-specified mixture and mesh of temperature and density.

The self-consistent set of photoionization and electron-ion recombination rate coefficients (for individual states and total) are available from the Ohio State University website: http://www.astronomy.ohio-state.edu/ pradhan.

\section{References}

Cunto, W., Mendoza, C., Ochsenbein, F., Zeippen, C.J, 1993, A\&A, 275, L5

Hummer, D.G., Berrington, K.A., Eissner, W., Pradhan, A.K., Saraph, H.E., \& Tully, J.A. 1993, A\&A, 279, 298

Seaton, M.J. 1997,MNRAS, 289, 700

Seaton, M.J. 1999,MNRAS, 307, 1008

Seaton, M.J., Yu, Y., Mihalas, D., Pradhan, A.K. 1994, MNRAS 266. 805

The Opacity Project 1 and 2, IOPP, Bristol $(1995,1996)$ 